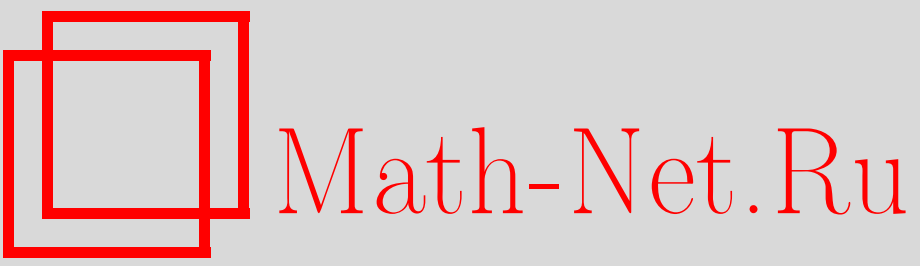

В. Г. Кадышевский, Г. А. Кравцова, В. Н. Родионов, Распад квазистационарных состояний нерелятивистских квантовых систем в интенсивном электромагнитном поле, ТМФ, 2002, том 130, номер 2, 275-286

DOI: https://doi.org/10.4213/tmf302

Использование Общероссийского математического портала Math-Net.Ru подразумевает, что вы прочитали и согласны с пользовательским соглашением

http://www.mathnet.ru/rus/agreement

Параметры загрузки:

IP : 54.162 .127 .20

26 апреля 2023 г., 05:29:13 
ТЕОРЕТИЧЕСКАЯ

И МАТЕМАТИЧЕСКАЯ

ФИЗИКА

Том 130, № 2

февраль, 2002

(С) 2002 г. В. Г. Кадышевский* , Г. А. Кравцова ${ }^{\dagger}$, В.Н. Родионов ${ }^{\ddagger}$

\title{
РАСПАД КВАЗИСТАЦИОНАРНЫХ СОСТОЯНИЙ НЕРЕЛЯТИВИСТСКИХ КВАНТОВЫХ СИСТЕМ В ИНТЕНСИВНОМ ЭЛЕКТРОМАГНИТНОМ ПОЛЕ
}

\begin{abstract}
Рассмотрено влияние интенсивных электромагнитных полей на формирование и распад квазистационарных состояний различных квантовых систем. На основе метода аналитического продолжения из выражений для вероятности процессов во внешнем электромагнитном поле получены нелинейные уравнения, определяющие комплексные значения энергии. Приведены асимптотические разложения, описывающие действие слабых и сильных электромагнитных полей. Отмечено, что существенное сокращение расстояний, определяюших формирование процессов в сверхсильных полях, может приводить к эффектам с характерным масштабом длины, свойственным формализму модифицированной КЭД - "КЭД с фундаментальной массой”.
\end{abstract}

\section{1. ВВЕДЕНИЕ}

Как известно, отличительные особенности вакуумных пороговых процессов прежде всего связаны с характерными энергетическими зависимостями вероятностей и сечений, которые наблюдаются при малых энергиях [1]. Эти зависимости полностью определяются видом волновых функций медленных частиц в состояниях с определенным орбитальным моментом и условиями конечности волновых функций системы. Сушественные изменения энергетических зависимостей могут наблюдаться в случаях, когда реакции указанного типа происходят во внешнем электромагнитном поле при участии в них заряженных частиш. Подобные процессы соответствуют, например, фотопоглошению непроводящими кристаллами, обусловленному разрешенными переходами, фотообразованию электрон-позитронных пар одинаково поляризованными фотонами, а также явлению ионизации систем типа атомов и ионов в электромагнитном поле.

\footnotetext{
* Объединенный институт ядерных исследований, Дубна, Московская обл., Россия

${ }^{\dagger}$ Московский государственный университет, Москва, Россия

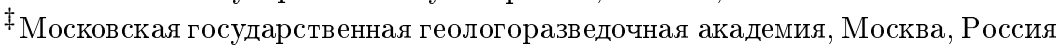


Возможность спонтанного перехода квантовой системы из определенного состояния в более низкие энергетические состояния приводит к тому, что такие системы нельзя рассматривать как строго стационарные. С формальной точки зрения квазистационарные состояния можно характеризовать как состояния с комплексной энергией. Следует также напомнить, что даже слабое электромагнитное поле способно изменить форму потенциального барьера и превратить финитную область движения электронов в инфинитную. Тем самым можно утверждать, что влияние поля, строго говоря, проявляется не только в изменении энергетического спектра системы, но и приводит к отличной от нуля вероятности ее распада (ионизация атомов под действием электрического поля, фотоэффект при энергии фотонов меньше ширины запрешенной зоны во внешнем поле, рождение электрон-позитронных пар двумя фотонами при любой их энергии и т.п.). В этом смысле включение в рассмотрение интенсивных электромагнитных полей с самого начала приводит к необходимости развивать подход, в котором состояния систем во внешнем поле рассматриваются как состояния с комплексными значениями энергии.

В работе [2] было рассмотрено взаимодействие частиц с суммарным орбитальным моментом равным нулю во внешнем поле довольно общего вида. В настояшей работе мы рассмотрим распад квазистационарных состояний ряда квантовых систем под действием интенсивного постоянного скрешенного электромагнитного поля $(\vec{E} \perp \vec{H},|E|=$ $|H|=F)$ вблизи порога соответствующих реакций в отсутствие поля. При этом мы не будем предполагать, что значения суммарного орбитального момента рассматриваемых систем равны нулю. Подобные явления могут наблюдаться, например, в полупроводниках при запрешенных переходах электронов между валентной зоной и зоной проводимости. Сюда же можно отнести и реакции фотообразования электрон-позитронных пар фотонами с различной поляризацией, а также явления фотоэффекта, связанные с распадом как атомных структур, так и некоторых ядерных систем (например, процесс фоторасщепления дейтрона).

Вероятности ряда процессов в постоянном интенсивном скрешенном электромагнитном поле представимы в виде однократных интегралов [2], [3]. Эти представления могут быть использованы для получения выражений, определяющих комплексные значения энергии, вещественные части которых соответствуют сдвигу уровней под влиянием поля, а мнимые - ширине уровней. Регулярное аналитическое продолжение этих интегральных представлений в комплексную плоскость обеспечивается построением дисперсионных соотношений с одним вычитанием [2]. Константой вычитания служит вероятность соответствуюшего процесса в отсутствие внешнего поля.

\section{2. УРАВНЕНИЯ ДЛЯ КОМПЛЕКСНЫХ ЗНАЧЕНИЙ ЭНЕРГИИ ВО ВНЕШНЕМ ПОЛЕ}

Для реакций, характеризуемых нулевым орбитальным моментом и протекающих в постоянном скрещенном поле произвольной интенсивности, можно получить нелинейное уравнение, определяюшее интересующую нас величину комплексной энергии $I$, нормированную на массу электрона. Следуя работе [2], запишем это уравнение в виде ин- 
тегральной зависимости от невозмущенной энергии $I_{0}{ }^{1)}$ :

$$
\varepsilon_{F}=\varepsilon_{0}+\frac{e^{-i \pi / 4}}{2 \sqrt{\pi}}\left(\frac{e F}{m^{2}}\right)^{1 / 3} f_{2}(z)
$$

где вместо энергий введены параметры $\varepsilon_{F}=\sqrt{-2 I}, \varepsilon_{0}=\sqrt{-2 I_{0}}$. Для функции $f_{2}(z)$ справедливо представление

$$
f_{2}(z)=\int_{0}^{\infty} \frac{d x}{x^{3 / 2}} e^{-i x z}\left(e^{-i x^{3} / 3}-1\right)
$$

а влияние поля проявляется через величины

$$
z=-2 I_{0}\left(\frac{m^{2}}{e F}\right)^{2 / 3}, \quad|z|=\frac{1}{\chi^{2 / 3}}, \quad \chi=\frac{e F}{m^{2}\left|2 I_{0}\right|^{3 / 2}}
$$

Здесь и далее используется система единиц, в которой $\hbar=c=1$. Отметим, что в книге [4] приводится расчет интегрального представления комплексной энергии, основанньй на анализе решений уравнения Шредингера для случая вырывания электрона из $\delta$-ямы полем интенсивной электромагнитной волны. Приведенный там результат согласуется (после исправления ряда опечаток) с уравнением (1) в пределе, когда частота волны $\omega \rightarrow 0$.

При выключении поля $\chi \rightarrow 0$ величина $|z|$ стремится к $\infty$, а различие знаков энерговыделения в процессе при $I_{0}>0$ или $I_{0}<0$ приводит к разным пределам величины $z$, которые согласно (3) соответственно равны $-\infty$ или $\infty$. Как отмечалось выше, величина $\operatorname{Im} I$ связана с вероятностью распада квазистационарной системы во внешнем поле в единицу времени, а вешественная часть рассматриваемого выражения позволяет получить информацию о сдвиге энергий во внешнем поле. В отличие от метода теории возмушений, в котором величины $\operatorname{Re} I$ и $\operatorname{Im} I$ вычисляются независимо друг от друга, в рассматриваемом подходе эти характеристики определяются из одного и того же уравнения (1).

Легко видеть, что при выключении поля предел $z \rightarrow \infty$ соответствует нулевой вероятности распада системы, так как при значении $I_{0}<0$ без участия поля система стабильна. В области $z<0$ мнимая часть величины $\varepsilon_{F}(z)$ определяет вакуумную вероятность распада, которая при положительном энерговыделении отлична от нуля даже в отсутствие поля. Вещественная же часть величины $\varepsilon_{F}$ при положительных значениях $z$ в пределе $\chi \rightarrow 0$ стремится к $\varepsilon_{0}$. На отрицательной полуоси $z \operatorname{Re} \varepsilon_{F}(-\infty)$ обрашается в нуль.

\footnotetext{
1) В частности, $I_{0}=2 \omega / m-2$ в случае двухфотонного рождения электрон-позитронных пар с суммарной энергией $2 \omega ; I_{0}=\left(\omega-E_{\mathrm{g}}\right) / m$ для процесса фотопоглощения кристаллом фотона с энергией $\omega$ при ширине запрещенной зоны $E_{\mathrm{g}}$ и безразмерной энергии фотоионизации $I_{0}$.
} 
Нетрудно убедиться, что при запрещенных по моменту переходах уравнение, определяющее ширину и сдвиг энергетических уровней, представимо в форме, аналогичной (1). Действительно, рассмотрим выражение для вероятности в скрешенном поле $W_{5 / 2}(F)$, полученное в работе [5] в форме однократного интеграла:

$$
\frac{W_{5 / 2}(F)}{W_{5 / 2}(0)}=\frac{3}{8 \sqrt{\pi}} \frac{e^{3 i \pi / 4}}{z^{3 / 2}} \int_{-\infty}^{\infty} \frac{d x}{(x-i \epsilon)^{5 / 2}} e^{-i x z-i x^{3} / 3},
$$

где $W_{5 / 2}(0)$ - вероятность процесса в отсутствие поля. Особенности подынтегральной функции в (4) сдвинуты с действительной оси $x$ в верхнюю полуплоскость, и интеграл понимается в смысле последующего предельного перехода $\epsilon \rightarrow 0$.

Для получения аналитического продолжения интегрального представления функции (4), заданной на действительной оси, в комплексную плоскость переменной $z$ необходимо произвести вычитание некоторой константы из исходной функции. В качестве константы вычитания выбираем значения вакуумной вероятности соответствуюшего процесса

$$
\frac{W_{5 / 2}(z)}{W_{5 / 2}(0)}=1+\frac{3}{8 \sqrt{\pi}} \frac{e^{3 i \pi / 4}}{z^{3 / 2}} \int_{-\infty}^{\infty} \frac{d x}{x^{5 / 2}} e^{-i x z}\left(e^{-i x^{3} / 3}-1\right) .
$$

Используя стандартную процедуру [2], [6], интеграл в выражении (5) можно привести к виду, позволяющему записать его мнимую и реальную части как

$$
G_{1}(z)=-\frac{i}{2} \int_{-\infty}^{\infty} \epsilon(u) \tilde{g}(u) e^{-i z u} d u, \quad G_{2}(z)=\frac{1}{2} \int_{-\infty}^{\infty} \tilde{g}(u) e^{i z u} d u
$$

где

$$
\tilde{g}(u)=\left\{\begin{array}{ll}
g(u), & u>0, \\
g^{*}(-u), & u<0,
\end{array} \quad g(u)=2 \frac{e^{5 i / 4 \pi}}{u^{5 / 2}}\left(e^{-i u^{3} / 3}-1\right),\right.
$$

a $\epsilon(u)=\operatorname{sign} u$.

Из формул (6), в частности, следует, что функции $G_{1}(z)$ и $G_{2}(z)$ вещественны на действительной оси $z$ и представимы в виде

$$
\begin{aligned}
& G_{1}(z)=\frac{1}{2 i} \int_{0}^{\infty}\left[g(u) e^{i z u}-g^{*}(u) e^{-i z u}\right] d u \\
& G_{2}(z)=\frac{1}{2} \int_{0}^{\infty}\left[g(u) e^{i z u}+g^{*}(u) e^{-i z u}\right] d u .
\end{aligned}
$$

Таким образом, функции $G_{1}, G_{2}$ определяют регулярную в нижней полуплоскости $z$ функцию

$$
G(z)=G_{2}(z)+i G_{1}(z)=\int_{0}^{\infty} g(u) e^{-i z u} d u
$$

вешественная и мнимая части которой на действительной оси $z$ связаны дисперсионным соотношением

$$
G_{1}\left(z_{0}\right)=-\frac{1}{\pi} \mathrm{P} \int_{-\infty}^{\infty} \frac{G_{2}(z)}{z-z_{0}} d z,
$$


где $\mathrm{P} \int_{-\infty}^{\infty}$ понимается как интеграл в смысле главного значения. Результатом проведения описанной процедуры является уравнение

$$
\varepsilon_{F}^{3}=\varepsilon_{0}^{3}+\frac{3 e^{5 i \pi / 4}}{4 \sqrt{\pi}} \frac{e F}{m^{2}} f_{3}(z),
$$

где

$$
f_{3}(z)=\int_{0}^{\infty} \frac{d x}{x^{5 / 2}} e^{-i x z}\left(e^{-i x^{3} / 3}-1\right) .
$$

Интегралы (2), (11) можно представить как линейные комбинации интегралов Меллина-Бернса, которые, в свою очередь, выражаются через специальные функции гипергеометрического типа:

$$
\begin{aligned}
f_{2}(z)= & \frac{1}{6}\left\{-\frac{8 \cdot 3^{5 / 6} i^{1 / 6} \pi}{\Gamma(1 / 6)}{ }_{1} F_{2}\left[-\frac{1}{6} ;\left\{\frac{1}{3}, \frac{2}{3}\right\}, \frac{z^{3}}{9}\right]+\right. \\
& +\frac{1}{\sqrt{\pi}}\left(12 \pi \sqrt{i z}-6^{2 / 3} i^{5 / 6} z \Gamma^{2}\left(\frac{1}{3}\right)\right){ }_{1} F_{2}\left[\frac{1}{6} ;\left\{\frac{2}{3}, \frac{4}{3}\right\}, \frac{z^{3}}{9}\right]+ \\
& \left.+\sqrt{3} i^{3 / 2} \pi z^{2}{ }_{1} F_{2}\left[\left\{\frac{1}{2}\right\} ;\left\{\frac{4}{3}, \frac{5}{3}\right\}, \frac{z^{3}}{9}\right]\right\}, \\
f_{3}(z)= & -\frac{4}{3} \sqrt{\pi}(i z)^{3 / 2}-\frac{2 \sqrt{i \pi / 3}}{3}{ }_{1} F_{2}\left[-\frac{1}{2} ;\left\{\frac{1}{3}, \frac{2}{3}\right\}, \frac{z^{3}}{9}\right]+ \\
& +\frac{6^{1 / 3} i^{7 / 6} z \Gamma^{2}(2 / 3)}{\sqrt{\pi}}{ }_{1} F_{2}\left[-\frac{1}{6} ;\left\{\frac{2}{3}, \frac{4}{3}\right\}, \frac{z^{3}}{9}\right]+ \\
& +3^{1 / 6} i^{11 / 6} z^{2} \Gamma\left(\frac{7}{6}\right){ }_{1} F_{2}\left[\frac{1}{6} ;\left\{\frac{4}{3}, \frac{5}{3}\right\}, \frac{z^{3}}{9}\right] .
\end{aligned}
$$

Выражения (12), (13) явным образом определяют нелинейные зависимости сдвига энергетических уровней и их ширины от параметра интенсивности поля $F$. Отметим, что содержащиеся в них гипергеометрические функции ${ }_{1} F_{2}$ табулированы и позволяют построить граффики величин $\operatorname{Re} \varepsilon_{F}$ и $\operatorname{Im} \varepsilon_{F}$ как функций параметра $z$. На рис. 1 и 2 приведены явные зависимости для функции $f_{2}(z)$, отвечаюшие значению интенсивности поля $e F / m^{2}=10^{-6}$. Легко видеть, что при $z<0$ у величин $\operatorname{Re}\left\{e^{-i \pi / 4} f_{2}(z)\right\}$ и $\operatorname{Im}\left\{e^{-i \pi / 4} f_{2}(z)\right\}$ наблюдаются осцилляции, а при $z>0$ - экспоненциальное убывание. Поведение реальной и мнимой частей функции $f_{3}(z)$ является аналогичным. Таким образом, в области $z \sim 0$, что соответствует значениям $I_{0} \sim 0$, функции $f_{2}(z)$ и $f_{3}(z)$ сглаживают ступенчатые зависимости характеристик в отсутствие поля.

Зависимости для мнимой и вешественной составляюших $\varepsilon_{F}(z)$ представлены на рис. 3 и 4. Построенные кривые соответствуют значениям $\varepsilon_{F}$ в поле, нормированным на $\varepsilon_{0}$ в вакууме. Таким образом, особенности в нуле имеют вакуумное происхождение и отвечают пределу $\varepsilon_{0} \rightarrow 0$ при $z \rightarrow 0$. Любопытно, что кривые на рис. 1 и 2 полностью повторяют ход так называемых функций электропоглошения продольного и поперечного типов, возникающих в интенсивном электрическом поле [7].

Для проведения численного анализа вещественных и мнимых частей $\varepsilon_{F}$ в постоянном поле интегралы (2), (11) можно выразить через билинейные комбинации функций 


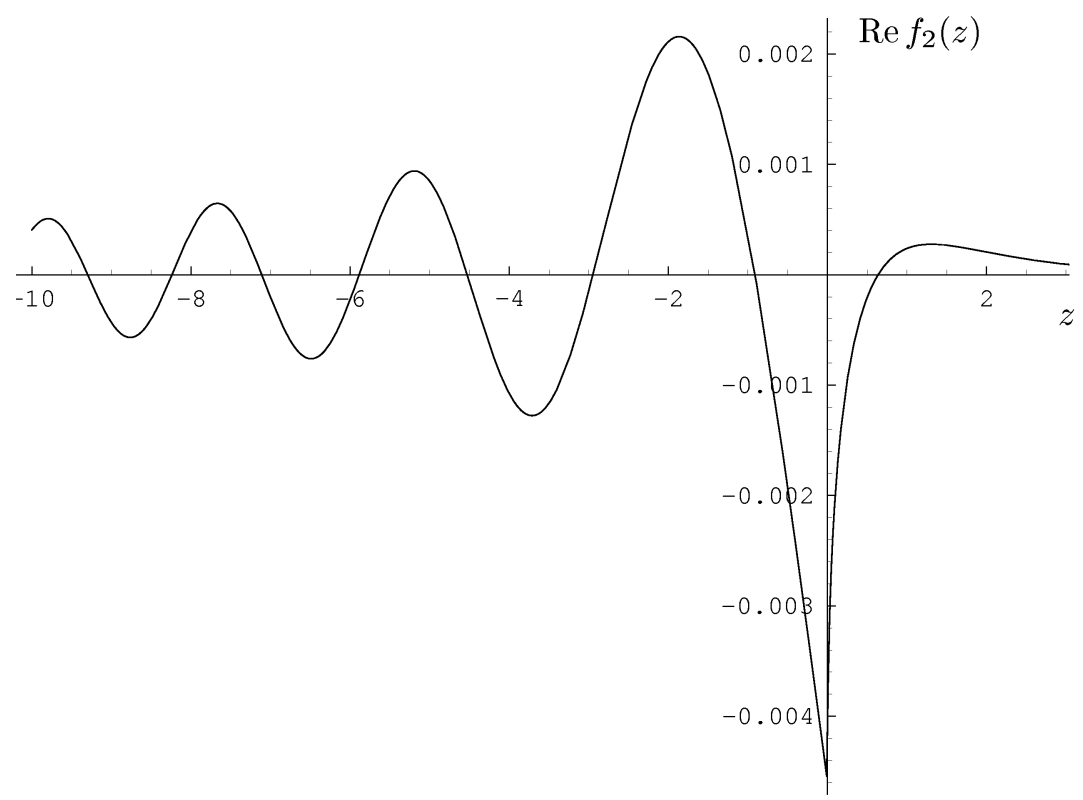

Рис. 1

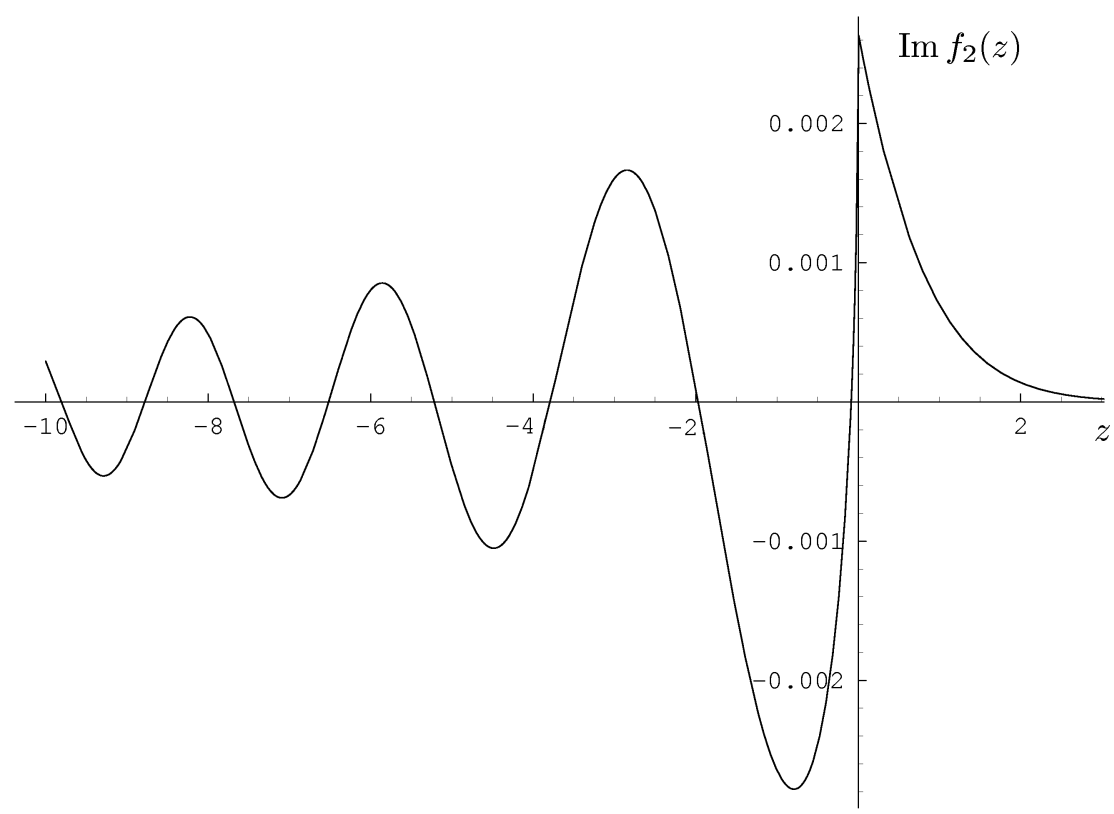

Рис. 2

Эйри. Для этого необходимо использовать ряд соотношений, вытекающих из представлений интегралов (2), (11), а также свойства функций Эйри $\mathrm{Ai} z, \mathrm{Bi} z$. В частности, если выполнить преобразования, аналогичные тем, которые приводили к соотношени- 


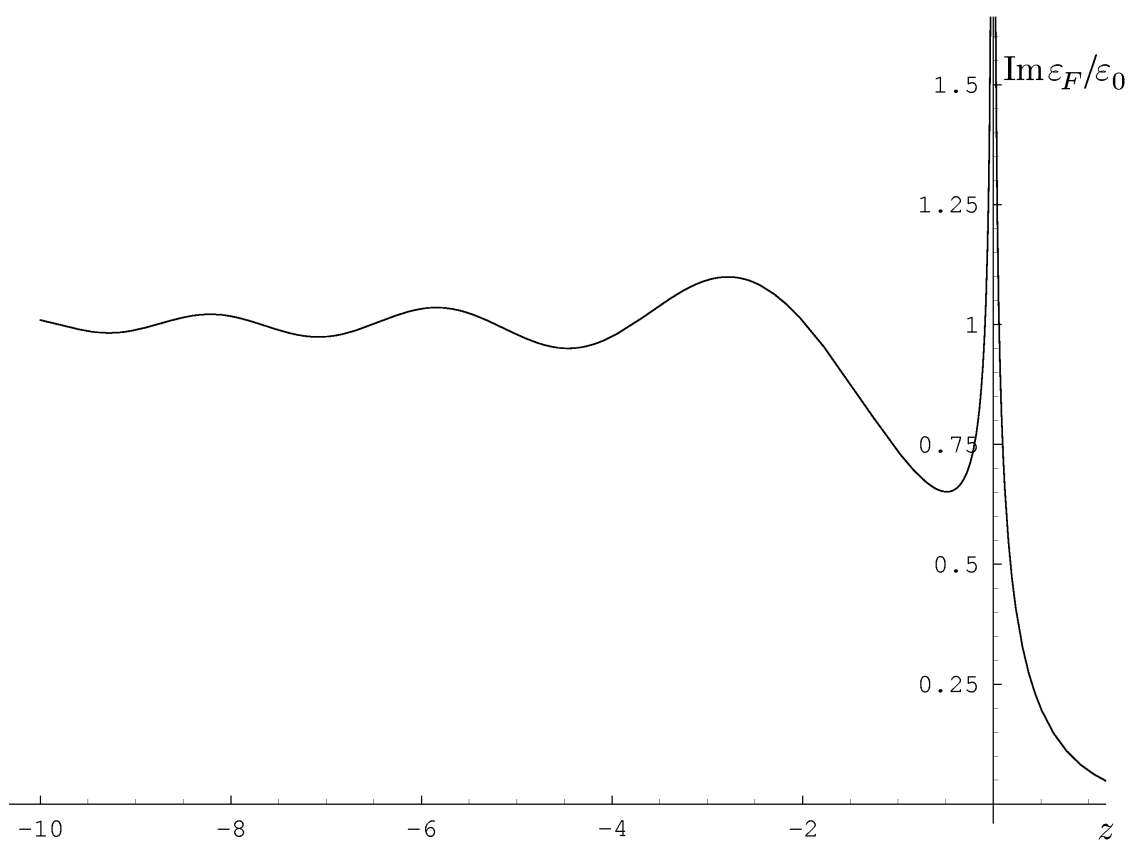

Рис. 3

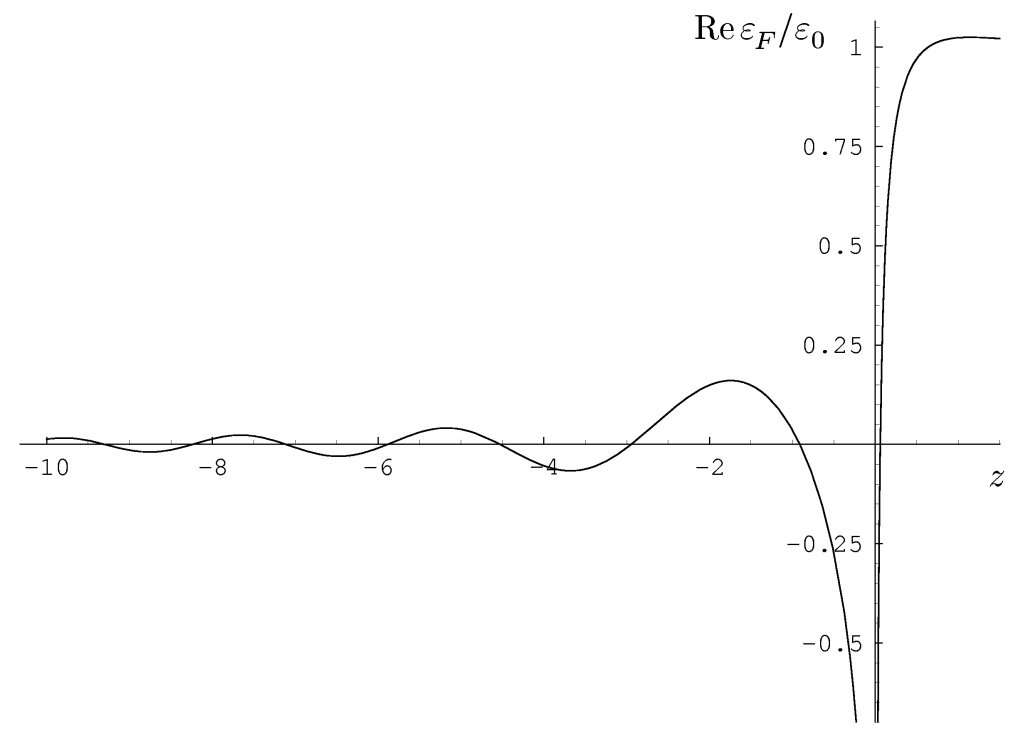

Рис. 4

ям (12), (13), и учесть известные выражения для квадратичных комбинаций функций Эйри [7], то можно получить соотношения, явным образом связываюшие гипергеомет- 
рические функции ${ }_{1} F_{2}$ с функциями $\mathrm{Ai}$ и $\mathrm{Bi}$ :

$$
\begin{aligned}
e^{-i \pi / 4} f_{1}(z)= & e^{i \pi / 4} \int_{0}^{\infty} d x \frac{e^{-i z x-i x^{3} / 3}}{\sqrt{x}}=\frac{\Gamma(1 / 6)}{3^{5 / 6}} e^{i \pi / 6}{ }_{1} F_{2}\left[\frac{1}{6} ;\left\{\frac{1}{3}, \frac{2}{3}\right\}, \frac{z^{3}}{9}\right]- \\
& -z \sqrt{\frac{\pi}{3}} e^{i \pi / 2}{ }_{1} F_{2}\left[\frac{1}{2} ;\left\{\frac{2}{3}, \frac{4}{3}\right\}, \frac{z^{3}}{9}\right]+ \\
& +\frac{z^{2} \Gamma(5 / 6)}{2 \cdot 3^{1 / 6}} e^{5 i \pi / 6}{ }_{1} F_{2}\left[\frac{5}{6} ;\left\{\frac{4}{3}, \frac{5}{3}\right\}, \frac{z^{3}}{9}\right]= \\
= & \frac{2^{2 / 3}}{\sqrt{\pi}} \operatorname{Ai}\left(\frac{z}{2^{2 / 3}}\right) \operatorname{Bi}\left(\frac{z}{2^{2 / 3}}\right)+i \frac{2^{2 / 3}}{\sqrt{\pi}} \operatorname{Ai}^{2}\left(\frac{z}{2^{2 / 3}}\right) .
\end{aligned}
$$

После ряда преобразований для интегралов рассматриваемого типа можно получить

$$
\begin{aligned}
f_{2}(z)= & 2 \sqrt{\pi} e^{i \pi \operatorname{sign} z / 4}|z|^{1 / 2}-\frac{2 e^{i \pi / 4}}{\sqrt{\pi}} \int_{0}^{\infty} d x x^{1 / 2} \mathrm{Ai}(z-x)+ \\
& +\frac{2 e^{3 i \pi / 4}}{\sqrt{\pi}} \int_{0}^{\infty} d x x^{1 / 2} \operatorname{Ai}(z+x) .
\end{aligned}
$$

Выделение действительной и мнимой частей в выражении (15) приводит к результатам

$$
\begin{aligned}
& \operatorname{Im}\left[e^{-i \pi / 4} f_{2}(z)\right]=-2 \sqrt{\pi}(-z)^{1 / 2} \theta(-z)+\frac{2}{\sqrt{\pi}} \int_{0}^{\infty} d x x^{1 / 2} \operatorname{Ai}(z+x), \\
& \operatorname{Re}\left[e^{-i \pi / 4} f_{2}(z)\right]=2 \sqrt{\pi} z^{1 / 2} \theta(z)-\frac{2}{\sqrt{\pi}} \int_{0}^{\infty} d x x^{1 / 2} \operatorname{Ai}(z-x),
\end{aligned}
$$

где

$$
\theta(z)= \begin{cases}1, & z>0 \\ 0, & z<0\end{cases}
$$

Выполняя аналогичные преобразования для $f_{3}$, получим представление

$$
\begin{aligned}
f_{3}(z)= & -\frac{4 \sqrt{\pi}}{3} e^{3 i \pi \operatorname{sign} z / 4}|z|^{3 / 2}+\frac{4 e^{3 i \pi / 4}}{3 \sqrt{\pi}} \int_{0}^{\infty} d x x^{3 / 2} \mathrm{Ai}(z-x)+ \\
& +\frac{4 e^{5 i \pi / 4}}{3 \sqrt{\pi}} \int_{0}^{\infty} d x x^{3 / 2} \operatorname{Ai}(z+x),
\end{aligned}
$$

откуда следует

$$
\begin{aligned}
& \operatorname{Im}\left[e^{-3 i \pi / 4} f_{3}(z)\right]=-\frac{4 \sqrt{\pi}}{3}(-z)^{3 / 2} \theta(-z)+\frac{4}{3 \sqrt{\pi}} \int_{0}^{\infty} d x x^{3 / 2} \operatorname{Ai}(z+x), \\
& \operatorname{Re}\left[e^{-3 i \pi / 4} f_{3}(z)\right]=-\frac{4 \sqrt{\pi}}{3} z^{3 / 2} \theta(z)+\frac{4}{3 \sqrt{\pi}} \int_{0}^{\infty} d x x^{3 / 2} \operatorname{Ai}(z-x) .
\end{aligned}
$$

Интегралы (16), (17) и (19), (20) выражаются через произведения функций Эйри, если воспользоваться соотношением (14) и применить легко проверяемую формулу

$$
\frac{(\mp 1)^{n}}{2^{2 / 3}} \int_{0}^{\infty} d x x^{n-1 / 2} \operatorname{Ai}(z \mp x)=\left(\frac{d^{2}}{d z^{2}}-z\right)^{n} \times\left\{\begin{array}{l}
\mathrm{Ai}\left(\frac{z}{2^{2 / 3}}\right) \mathrm{Bi}\left(\frac{z}{2^{2 / 3}}\right), \\
\mathrm{Ai}^{2}\left(\frac{z}{2^{2 / 3}}\right),
\end{array}\right.
$$


обобщаюшую аналогичное соотношение для $\mathrm{Ai}^{2}$ в одной из работ [3]. Таким образом, после некоторых преобразований получим выражения

$$
\begin{aligned}
& \int_{0}^{\infty} d x x^{1 / 2} \operatorname{Ai}(z+x)=-\frac{z}{2^{1 / 3}} \operatorname{Ai}^{2}\left(\frac{z}{2^{2 / 3}}\right)+2^{1 / 3} \operatorname{Ai}^{\prime 2}\left(\frac{z}{2^{2 / 3}}\right), \\
& -\int_{0}^{\infty} d x x^{1 / 2} \operatorname{Ai}(z-x)=-\frac{z}{2^{1 / 3}} \operatorname{Ai}\left(\frac{z}{2^{2 / 3}}\right) \operatorname{Bi}\left(\frac{z}{2^{2 / 3}}\right)+ \\
& +2^{1 / 3} \mathrm{Ai}^{\prime}\left(\frac{z}{2^{2 / 3}}\right) \mathrm{Bi}^{\prime}\left(\frac{z}{2^{2 / 3}}\right) \text {, } \\
& \int_{0}^{\infty} d x x^{3 / 2} \operatorname{Ai}(z+x)=\frac{z^{2}}{2^{1 / 3}} \operatorname{Ai}^{2}\left(\frac{z}{2^{2 / 3}}\right)-\operatorname{Ai}\left(\frac{z}{2^{2 / 3}}\right) \operatorname{Ai}^{\prime}\left(\frac{z}{2^{2 / 3}}\right)- \\
& -z 2^{1 / 3} \mathrm{Ai}^{\prime 2}\left(\frac{z}{2^{2 / 3}}\right) \text {, } \\
& \int_{0}^{\infty} d x x^{3 / 2} \operatorname{Ai}(z-x)=\frac{z^{2}}{2^{1 / 3}} \operatorname{Ai}\left(\frac{z}{2^{2 / 3}}\right) \operatorname{Bi}\left(\frac{z}{2^{2 / 3}}\right)- \\
& -\frac{1}{2}\left[\operatorname{Ai}\left(\frac{z}{2^{2 / 3}}\right) \operatorname{Bi}^{\prime}\left(\frac{z}{2^{2 / 3}}\right)+\operatorname{Ai}^{\prime}\left(\frac{z}{2^{2 / 3}}\right) \operatorname{Bi}\left(\frac{z}{2^{2 / 3}}\right)\right]- \\
& -z 2^{1 / 3} \mathrm{Ai}^{\prime}\left(\frac{z}{2^{2 / 3}}\right) \mathrm{Bi}^{\prime}\left(\frac{z}{2^{2 / 3}}\right) \text {, }
\end{aligned}
$$

где $\mathrm{Ai}^{\prime}$ и $\mathrm{Bi}^{\prime}$ - производные соответствуюших функций по аргументу. Полученные соотношения позволяют легко установить, что мнимая часть выражения (1) содержит результаты, определяющие вероятности процессов с нулевым суммарным орбитальным моментом [3], [5], [8]. Аналогично из (10) можно получить вероятности процессов при значении орбитального момента (выраженного в единицах постоянной Планка $\hbar$ ) равном 2 [5]. Вешественные части (17), (20) и (22), (24) позволяют получить информацию об энергетических сдвигах, обусловленных действием внешнего поля.

\section{3. АСИМПТОТИЧЕСКИЕ ЗНАЧЕНИЯ ШИРИНЫ И СДВИГА ЭНЕРГЕТИЧЕСКИХ УРОВНЕЙ}

Если поле слабо, то параметр $\chi=\left(e F / m^{2}\right)(2|I|)^{3 / 2} \ll 1$ и величина $|z| \gg 1$. Как отмечалось вьше, в зависимости от знака $I_{0}$ аргумент функций Эйри принимает большие положительные или отрицательные значения. В частности, используя асимптотики для билинейных комбинаций функций Эйри и их производных (см. [2]) при $z \gg 1\left(I_{0}<0\right)$, из выражения (1) получаем

$$
\begin{aligned}
& \operatorname{Im} \sqrt{-2 I}=\sqrt{-2 I_{0}} \frac{\chi}{4} e^{-2 /(3 \chi)}\left(1-\frac{17}{12} \chi+\frac{1225}{288} \chi^{2}\right), \\
& \operatorname{Re} \sqrt{-2 I}=\sqrt{-2 I_{0}}\left(1+\frac{1}{8} \chi^{2}+\frac{105}{128} \chi^{4}\right),
\end{aligned}
$$

откуда следует, что мнимая часть определяется экспоненциальной зависимостью, а вешественная содержит квадратичные поправки к вакуумному значению величины $\varepsilon_{0}$. 
В случае $I_{0}>0$ аргумент функций Эйри отрицателен, и при $\chi \ll 1$ величина $-z \gg 1$. В этом пределе для мнимой и вешественной частей выражения (1) соответственно имеем

$$
\begin{aligned}
& \operatorname{Im} \sqrt{-2 I}=\sqrt{2 I_{0}}\left\{1-\frac{\chi}{2} \cos \frac{2}{3 \chi}+\frac{\chi^{2}}{24}\left[3-17 \sin \frac{2}{3 \chi}\right]+\frac{1225 \chi^{3}}{576} \cos \frac{2}{3 \chi}\right\} \\
& \operatorname{Re} \sqrt{-2 I}=\sqrt{2 I_{0}}\left\{\frac{\chi}{2} \sin \frac{2}{3 \chi}-\chi^{2} \frac{17}{24} \cos \frac{2}{3 \chi}-\frac{1225}{576} \chi^{3} \sin \frac{2}{3 \chi}\right\} .
\end{aligned}
$$

Из выражения (27) видно, что полевые поправки к мнимой части содержат как "монотонный”, так и “осциллирующий” вклады (см. [9]). Действительная часть (28) имеет лишь осцилляционную зависимость. Отметим, что первые члены разложений (25)-(28) совпадают с аналогичными результатами работы [2], полученными другим способом.

Оценивая величину (10) в том же пределе слабого поля $z \gg 1$ при $I_{0}<0$, получим

$$
\begin{aligned}
& \operatorname{Im}(-2 I)^{3 / 2}=\left(-2 I_{0}\right)^{3 / 2} \frac{3 \chi^{2}}{8} e^{-2 /(3 \chi)}\left(1-\frac{35}{12} \chi+\frac{3745}{288} \chi^{2}\right), \\
& \operatorname{Re}(-2 I)^{3 / 2}=\left(-2 I_{0}\right)^{3 / 2}\left(1+\frac{\chi^{2}}{8}+\frac{35}{128} \chi^{4}\right) .
\end{aligned}
$$

В случае $I_{0}>0$ в пределе слабого поля параметр $-z \gg 1$, и для мнимой и действительной частей $\varepsilon_{F}^{3}$ соответственно имеем

$$
\begin{aligned}
\operatorname{Im}(-2 I)^{3 / 2}= & \left(2 I_{0}\right)^{3 / 2}\left\{1-\frac{\chi^{2}}{8}+\frac{35}{128} \chi^{4}+\right. \\
& \left.+\left(-\frac{3}{4} \chi^{2}+\frac{3745}{384} \chi^{4}\right) \sin \frac{2}{3 \chi}+\frac{35}{16} \chi^{3} \cos \frac{2}{3 \chi}\right\}, \\
\operatorname{Re}(-2 I)^{3 / 2}= & \left(2 I_{0}\right)^{3 / 2}\left\{\left(-\frac{3}{4} \chi^{2}+\frac{3745}{384} \chi^{4}\right) \cos \frac{2}{3 \chi}-\frac{35}{16} \chi^{3} \sin \frac{2}{3 \chi}\right\} .
\end{aligned}
$$

Формулы (29)-(32) подтверждают основные выводы, сделанные при анализе выражений (25)-(28), однако в них экспоненциальный и осциллирующие вклады содержат дополнительньй множитель $\chi \ll 1$. Это приводит к их относительному ослаблению по сравнению с аналогичными вкладами (25), (27), (28).

В пределе сильного поля, т.е. при $\chi \gg 1$, аргумент функций Эйри мал: $|z| \ll 1$, и характер энергетических зависимостей сушественно меняется. Для процессов, описываемых уравнением (1), основные результаты приводились в работе [2]. Оценивая в этом пределе выражение (11) и оставляя лишш главные члены разложений при $z \ll 1$, нетрудно получить

$$
\operatorname{Re} I=-\frac{1}{3^{1 / 3} 2^{8 / 3}}\left(\frac{e F}{m^{2}}\right)^{2 / 3}, \quad \operatorname{Im} I=-\frac{3^{1 / 6}}{2^{8 / 3}}\left(\frac{e F}{m^{2}}\right)^{2 / 3} .
$$

Из формул (33), в частности, следует, что при значениях интенсивности поля, больших по сравнению с энерговыделением в распаде $e F / m^{2} \gg\left|2 I_{0}\right|^{3 / 2}$, ширина уровня и его сдвиг, так же как и в случае реакций с нулевым орбитальным моментом, пропорциональны $\left(e F / m^{2}\right)^{2 / 3}$. При этом реальная и мнимая части энергии по-прежнему отличаются только числовым множителем $\sqrt{3}$. 


\section{4. ЗАКЛЮЧИТЕЛЬНЫЕ ЗАМЕЧАНИЯ И ОБСУЖДЕНИЕ}

Существенная особенность выражений (1), (10) на бесконечности по переменной, пропорциональной невозмущенной энергии и обратно пропорциональной заряду и внешнему полю, соответствует появлению нестабильности квантовых систем в поле. Действительно, под влиянием поля возникает вероятность прохождения электрона через потенциальный барьер при любом значении энергии частицы. Характер существенной особенности вида $e^{-z^{3 / 2}}$ тесно связан с возникающим в этом случае подбарьерным туннелированием.

Неаналитичность точных выражений по внешнему полю в точке его выключения $\chi \rightarrow 0$ хорошо известна. Достаточно напомнить, что аналогичные зависимости возникают в задаче об ионизации атомов в электрическом поле, а также при анализе эффекта Штарка [1]. Выражения того же вида получаются и в случае изучения особенностей по динамической переменной и квадрату импульса для массового и поляризационного операторов [3], [8] в постоянном скрешенном поле.

В области сильного поля, т.е. когда параметр $\chi \gg 1$, сравнение полученных значений для ширины уровня энергии и его сдвига со значениями реальных и мнимых частей радиационных поправок к массам электрона и фотона во внешнем поле [3], [8] обнаруживает их глубокую аналогию. Она выражается в том, что рост указанных характеристик с увеличением интенсивности поля, по существу, определяется одной и той же функциональной зависимостью от полевого параметра $\sim\left(e F / m^{2}\right)^{2 / 3}$. Отличия сводятся лишь к разным числовым множителям, причем относительный множитель, связывающий реальные и мнимые части, оказывается одним и тем же в обоих случаях. На эту универсальность указывают и результаты, полученные нами для процессов с отличным от нуля значением орбитального момента. Причем важно подчеркнуть, что отмеченные аналогии имеют место, несмотря на то что нами рассмотрено нерелятивистское приближение, в то время как в работах [3], [8] (см. также многочисленные работы, цитированные там) развивался релятивистский подход.

Это можно объяснить тем, что рассмотренная нами область значений $\chi \gg 1$ непосредственно смыкается с областью сверхсильных полей $e F \gg m^{2}$, в которой изучаемые характеристики полностью определяются действием внешнего поля. Необходимо подчеркнуть, что здесь утрачивается исключительность масштаба не только де-бройлевской, но и комптоновской длины волны электрона $\left(\lambda_{c}\right)$, так как характерным параметром оказывается так называемая магнитная длина $r_{H}=\lambda_{\mathrm{c}}\left(m^{2} / e F\right)^{1 / 2} \ll \lambda_{\mathrm{c}}[10]$. Дальнейшее повышение интенсивности поля уже не может изменить этот масштаб в рамках традиционной квантовой электродинамики (КЭД). Однако существенное сокращение расстояний, характеризующих формирование процессов в таких полях, может приводить к эффектам, определяемым уже другим масштабом длины, свойственным формализму модифицированной КЭД.

Последовательное изучение подобных явлений может быть проведено, например, в рамках новой теории - "КЭД с фундаментальной массой" [10]- [13]. В этом подходе основные эффекты, обусловленные действием интенсивного внешнего поля, определяются соотношением между двумя параметрами - фундаментальной и электромагнитной дли- 
нами [10].

Проведенное исследование показывает, что комплексные величины $\varepsilon_{F}$ и $\varepsilon_{F}^{3}$ имеют сушественные особенности на бесконечности и точки ветвления в нуле. Проводя разрез по отрицательной полуоси $z<0$, получаем функции $\varepsilon_{F}(z)$ и $\varepsilon_{F}^{3}(z)$, аналитические всюду на конечной плоскости, за исключением линии разреза. При $|z| \rightarrow \infty$ в секторе $-\pi<\arg z<\pi / 3$ полевые функции спадают, а в секторе $\pi / 3<\arg z<\pi$ они экспоненциально растут. В $\chi$-плоскости величины $\varepsilon_{F}$ и $\varepsilon_{F}^{3}$ имеют сушественные особенности и ветвление в точке $\chi=0$.

Из-за ветвления возникает неоднозначность энергетических зависимостей от поля. Ветвление выделяет две области аналитичности, которые с учетом соотношения $\arg z=$ $-(2 / 3) \arg \chi$ определяются условиями

$$
-\frac{\pi}{2}<\arg \chi<\frac{3 \pi}{2}, \quad \frac{5 \pi}{2}<\arg \chi<\frac{9 \pi}{2} .
$$

Изменение $\arg z$ на $2 \pi$ приводит к переходу на соседний лист и изменяет знак $\sqrt{z}$.

Заметим, что из-за сушественной особенности в точке $\chi=0 \quad(z \rightarrow \infty)$ предельные значения $\operatorname{Im} \varepsilon_{F}$ и $\operatorname{Im} \varepsilon_{F}^{3}$ в отсутствие поля обнаруживают экспоненциальные поправки $\sim e^{-2 z^{3 / 2} / 3}$, а реальные части имеют поправки, квадратичные по полю. При $z \rightarrow-\infty$ нулевые значения поправок достигаются как предел быстро осциллирующих функций с бесконечным числом их обращений в нуль. Свойства функции $f_{3}(z)$ во многом сходны со свойствами $f_{2}(z)$, однако из-за более высокой степенной зависимости монотонных вкладов $\sim z^{3}$ осцилляционное поведение $\varepsilon_{F}^{3}$ выражено слабее по сравнению с $\varepsilon_{F}$.

Благодарности. Работа выполнена при поддержке РФФИ (грант № 99-02-17936-а) и при частичном финансировании гранта поддержки ведуших научных школ № 00-1596046 и гранта Фонда фундаментальных исследований при С.-ПбГУ № Е00-3.1-457.

\section{Список литературы}

[1] Л. Д. Ландау, Е. М. Лифииц. Квантовая механика. М.: Наука, 1989.

[2] В. Г. Кадышевский, В. Н. Родионов. ТМФ. 2000. Т. 125. № 3. С. 432.

[3] А. И. Никишов, В. И. Ритус. Тр. ФИАН. 1979. Т. 111. С. 84; 1986. Т. 168. С. 232.

[4] Н. Б. Делоне, В. П. Крайнов. Атом в сильном световом поле. М.: Атомиздат, 1978.

[5] В. Н. Родионов. ЖЭТФ. 1998. Т. 113. С. 21.

[6] Х. М. Нуссенцвейг. Причинность и дисперсионные соотношения. М.: Мир, 1976.

[7] D. E. Aspnes. Phys. Rev. 1966. V. 147. P. 554.

[8] И. М. Тернов, В. Р. Халилов, В. Н. Родионов. Взаимодействие заряженных частиц с сильным электромагнитным полем. М.: Изд-во МГУ, 1982.

[9] А. Е. Лобанов, А. Р. Муратов. ЖЭТФ. 1984. Т. 87. С. 1140.

[10] В. Г. Кадышевский, В. Н. Родионов. Электромагнитная длина и фундаментальная масса в электродинамике. В сб.: Тр. семинара "Симметрии и интегрируемые системы” . Ред. А. Н. Сисакян. Дубна: ОИЯИ, 1999. С. 103.

[11] В. Г. Кадьиевский. ЭЧАЯ. 1980. Т. 11. С. 5.

[12] V. G. Kadyshevsky, M. D. Mateev. Phys. Lett. B. 1981. V. 106. P. 139.

[13] В. Г. Кадышевский. ЭЧАЯ. 1998. Т. 29. С. 563.

Поступила в редакцию 11.VII.2001 г. 